\title{
Urinary bisphenol A concentration and glucose homeostasis in non-diabetic adults: a repeated-measures, longitudinal study
}

\author{
Bin Wang ${ }^{1,2,3} \cdot$ Mian $\mathrm{Li}^{1,2,3} \cdot$ Zhiyun Zhao ${ }^{1,2,3} \cdot$ Jieli Lu ${ }^{1,2,3} \cdot$ Yuhong Chen ${ }^{1,2,3} \cdot Y u^{X} u^{1,2,3} \cdot M^{2} X^{1,2,3} \cdot$ \\ Weiqing Wang ${ }^{1,2,3} \cdot$ Tiange Wang ${ }^{1,2,3} \cdot$ Yufang $\mathrm{Bi}^{1,2,3} \cdot$ Guang Ning ${ }^{1,2,3}$
}

Received: 4 February 2019 / Accepted: 18 April 2019 / Published online: 15 May 2019

(C) Springer-Verlag GmbH Germany, part of Springer Nature 2019

\begin{abstract}
Aims/hypothesis Bisphenol A (BPA) has been shown to be potentially associated with type 2 diabetes; however, there is little evidence associating BPA exposure with glucose metabolic outcomes prior to diabetes onset. We aimed to examine BPA exposure in relation to glucose homeostasis among non-diabetic individuals.

Methods This longitudinal cohort study comprised 2336 Chinese adults aged 40 years or above (62.8\% women) and free of diabetes at baseline in 2009, followed for 4 years. Urinary BPA and glucose metabolic traits including fasting plasma glucose (FPG), 2 h postload plasma glucose, fasting serum insulin, HOMA-IR and HOMA-B were measured at baseline and follow-up. Repeated-measures analysis was performed to evaluate associations of urinary BPA concentration with markers of glucose homeostasis.

Results After full adjustment for confounders including BMI, each tenfold increase in urinary BPA concentrations was associated with a $3.39 \%$ increase in FPG $(95 \%$ CI $2.24 \%, 4.55 \%)$ and an $11.6 \%$ decrease in HOMA-B (95\% CI $-15.8 \%,-7.18 \%)$ in women. The inverse association between urinary BPA and HOMA-B was more prominent among overweight or obese individuals (change $-13.7 \%$; $95 \%$ CI $-19.3 \%,-7.61 \%$ ) compared with those who were of normal weight (change $-6.74 \%$; $95 \%$ CI $-13.2 \%, 0.20 \%)\left(p_{\text {interaction }}=0.07\right)$. Moreover, the ORs of fasting hyperglycaemia and beta cell dysfunction corresponding to a tenfold increase in urinary BPA concentrations were 1.37 (95\% CI 1.10, 1.72) and 1.30 (95\% CI 1.02, 1.65) in women, respectively. No significant associations existed between urinary BPA and glucose metabolic markers in men.

Conclusions/interpretation Our findings suggest that exposure to BPA was independently associated with impaired glucose homeostasis before the development of diabetes in middle-aged and elderly women.
\end{abstract}

Keywords Bisphenol A · Glucose homeostasis · HOMA-B · Repeated measures · Type 2 diabetes

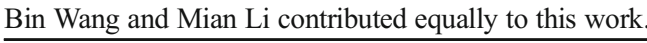

Electronic supplementary material The online version of this article (https://doi.org/10.1007/s00125-019-4898-x) contains peer-reviewed but unedited supplementary material, which is available to authorised users.

Yufang Bi

byf10784@rjh.com.cn

Tiange Wang

wtg@live.cn

1 State Key Laboratory of Medical Genomics, Key Laboratory for Endocrine and Metabolic Diseases of Ministry of Health, National Clinical Research Center for Metabolic Diseases, Rui-Jin Hospital, Shanghai Jiao Tong University School of Medicine, 197 Rui-Jin 2nd Road, Shanghai 200025, China

2 Department of Endocrine and Metabolic Diseases, Rui-Jin Hospital, Shanghai Jiao Tong University School of Medicine, Shanghai, China

3 Shanghai Institute of Endocrine and Metabolic Diseases, Rui-Jin Hospital, Shanghai Jiao Tong University School of Medicine, Shanghai, China

\section{Abbreviations}

BPA Bisphenol A

FPG Fasting plasma glucose

GAMM Generalised additive mixed models

IQR Interquartile range

PPG Post-load plasma glucose

\section{Introduction}

Type 2 diabetes, together with its related morbidity and mortality, has become a major public health crisis worldwide [1]. The increasing burden of type 2 diabetes is primarily attributed to unhealthy dietary habits and lifestyles [2]. In the past decade, bisphenol A (BPA), a well-documented endocrine disruptor, has been associated with the epidemics of obesity and diabetes [3-5]. BPA is a high-production-volume 


\section{Research in context}

\section{What is already known about this subject?}

- Bisphenol A (BPA), a ubiquitous endocrine-disrupting chemical, has been found to be associated with type 2 diabetes

- Less is known about the association in humans between BPA and glucose metabolic traits, and previous evidence has been limited by cross-sectional study design

\section{What is the key question?}

- Can BPA exposure at environmentally relevant levels affect glucose homeostasis before the development of diabetes?

\section{What are the new findings?}

- The associations between repeated measures of urinary BPA and markers of glucose homeostasis in non-diabetic adults were sex-dependent

- Urinary BPA concentrations were positively associated with fasting glucose levels and inversely associated with HOMA-B in women; the estimates for HOMA-B were stronger among overweight or obese individuals than among those who were of normal weight

- In women, urinary BPA concentrations were associated with an increased risk of fasting hyperglycaemia and beta cell dysfunction

\section{How might this impact on clinical practice in the foreseeable future?}

- Our results indicate that female exposure to BPA has detrimental effects on glucose homeostasis even before the onset of diabetes. As this is a potentially highly modifiable risk factor, such findings may have implications for developing intervention strategies against BPA exposure, especially among high-risk populations, to help prevent and alleviate the burden of diabetes

chemical that is extensively used in manufacturing of epoxy resins lining food and drinks containers and polycarbonate plastics in numerous consumer products [6]. BPA may leach from coatings and plastics, and this process can be accelerated under high heat, physical manipulation or repetitive use. Therefore, human exposure to BPA is widespread, primarily through dietary intake but also through drinking water, dermal exposure and inhalation [7]. Once ingested, BPA is rapidly and almost completely conjugated in phase II reactions to form the highly water-soluble metabolite; thus, urine is generally considered to be the most appropriate body fluid for assessment of BPA exposure [8]. Detectable concentrations of urinary BPA have been shown in diverse populations worldwide [9].

In addition to the well-known oestrogenic effects, experimental studies suggest that BPA may interfere with multiple pathways to initiate or exacerbate the development of diabetes, including dysregulation of glucose metabolism through impaired pancreatic beta cell function and insulin resistance, and inhibition of adiponectin release in adipocytes [10-12]. Mounting human data have reported the association between BPA and type 2 diabetes, but the results remain inconclusive [13-16]. More importantly, epidemiological studies have mostly examined diabetes risk as the outcome, and less is known about the link between BPA and glucose homeostasis.

Previous studies that assessed glucose metabolic traits in relation to BPA exposure have provided suggestive evidence for the adverse effects of BPA on glucose metabolism [17, 18]. However, these studies were limited by a cross-sectional design with single biomarker measurement in urine and blood samples collected at one time point. Therefore, these findings are likely to be partly biased due to measurement error or exposure misclassification, especially when the temporal variability of biomarker levels is high, as in the case of BPA [19]. This emphasises the necessity of using multiple measurements to obtain a more robust assessment of exposure levels.

Although impaired glucose homeostasis is predictive of subsequent diabetes and atherosclerotic cardiovascular disease, few studies to date have investigated whether BPA exposure can alter glucose homeostasis prior to diabetes onset. Our previous study indicated the potential effect of BPA exposure on deterioration in fasting plasma glucose (FPG) among high genetically predisposed Chinese adults without baseline diabetes [20]. To address the current knowledge gap, we further examined the associations between repeated measures of urinary BPA and markers of glucose homeostasis among a prospective cohort of middle-aged and elderly Chinese individuals without a history of diabetes.

\section{Methods}

Study population Participants in the present study were from an ongoing prospective cohort of Chinese adults in Shanghai, 
as reported previously $[16,20]$. Between June and July 2008 (phase I), 10,185 registered permanent residents aged $\geq 40$ years from Songnan Community participated in the screening examination for an investigation aimed to explore the effects of risk factors on type 2 diabetes and related chronic diseases. Participants were classified into three groups according to FPG levels: normal glucose regulation (FPG $<5.6 \mathrm{mmol} / \mathrm{l}$ and no history of diabetes); impaired glucose regulation (FPG 5.6 to $<7.0 \mathrm{mmol} / 1$ and no history of diabetes); diabetes (FPG $\geq 7.0 \mathrm{mmol} / 1$ or a history of diabetes). Between June and August 2009 (phase II), participants were randomly selected from these groups, in a ratio of 1.0 (diabetes) to 1.2 (impaired glucose regulation) to 1.44 (normal glucose regulation), to take part in a comprehensive survey comprising a detailed questionnaire, anthropometric measurement, a standard $75 \mathrm{~g}$ OGTT and blood and urine sampling. Individuals with lower glucose levels were oversampled because they were expected to have a lower participation rate than those with higher glucose levels. The phase II survey comprised 3455 participants with urine and blood samples.

Between March and May 2013, enrolled participants ( $n=$ 3455 ) were invited to complete a follow-up survey. Of the 2336 participants without baseline diabetes (1999 WHO criteria: $\mathrm{FPG} \geq 7.0 \mathrm{mmol} / \mathrm{l}$ or OGTT $2 \mathrm{~h}$ post-load plasma glucose $[\mathrm{PPG}] \geq 11.1 \mathrm{mmol} / 1$ or receiving glucose-lowering treatments), 19 had died and 588 failed to attend the followup visit; 21 with newly glucose-lowering medication use and three with missing urine or blood samples were excluded. Thus, our final sample comprised 2336 participants, of whom 1705 had both baseline and follow-up data, yielding a total of 4041 observations with metabolic markers and urinary BPA measurements available for analysis. A flow chart showing the study procedure is shown in electronic supplementary material (ESM) Fig. 1. The study protocol was approved by the Committee on Human Research at Rui-Jin Hospital, Shanghai Jiao Tong University School of Medicine. All study participants provided written informed consent.

BPA measurement Total (free and conjugated) BPA concentrations in morning spot urine samples collected at two time points were measured by a sensitive and selective LC-MS/MS at the Shanghai Institute of Materia Medica, Chinese Academy of Sciences. Details regarding the quantification of urinary BPA concentration have been reported elsewhere [16]. The calibration curve covered the concentrations ranging from $0.30 \mu \mathrm{g} / 1$ to $100 \mu \mathrm{g} / \mathrm{l}$ for BPA using weighted least-squares linear regression mode. Both the intra- and inter-day relative SDs were calculated based on quality control samples at low, moderate and high levels $(0.80 \mu \mathrm{g} / \mathrm{l}, 10.0 \mu \mathrm{g} / \mathrm{l}$ and $80.0 \mu \mathrm{g} / \mathrm{l})$. The average relative SDs were less than $8.7 \%$ and the accuracy ranged from $98.0 \%$ to $98.5 \%$. Furthermore, the inter-day relative error as determined from quality control samples was within $2.5 \%$. For BPA levels below the limit of detection
(LOD, $0.3 \mu \mathrm{g} / \mathrm{l}$ ), a value of LOD divided by 2 was assigned for analysis [21]. Urinary creatinine levels were measured with a clinical chemistry automated analyser (ADVIA-1650 Chemistry System; Bayer, Leverkusen, Germany).

Markers of glucose homeostasis At baseline and the follow-up visit, all participants underwent a standard $75 \mathrm{~g}$ OGTT after an overnight fast of more than $10 \mathrm{~h}$. Blood samples were obtained at $0 \mathrm{~h}$ and $2 \mathrm{~h}$ during the test. Laboratory tests measured FPG, OGTT $2 \mathrm{~h}$ PPG, fasting serum insulin, and lipid profiles comprising total cholesterol, HDL-cholesterol, LDLcholesterol and triacylglycerols. Plasma glucose was measured using the glucose oxidase method on an automated analyser (ADVIA-1650 Chemistry System; Bayer). Serum insulin was measured by electrochemiluminescence assay (Roche Diagnostics, Basel, Switzerland). Blood glucose and insulin were measured within $2 \mathrm{~h}$ after sample collection. Measurements of total cholesterol, HDL-cholesterol, LDLcholesterol and triacylglycerols were performed with an automated analyser (ADVIA-1650 Chemistry System; Bayer).

In the current study, fasting and postprandial hyperglycaemia were defined as FPG $\geq 5.6 \mathrm{mmol} / 1$ and OGTT $2 \mathrm{~h}$ PPG $\geq 7.8 \mathrm{mmol} / \mathrm{l}$, respectively [22]. The HOMA-IR was calculated using the formula: fasting insulin $(\mathrm{pmol} / \mathrm{l}) \times \mathrm{FPG}(\mathrm{mmol} / \mathrm{l}) /$ 156.3. Insulin resistance was defined as HOMA-IR $\geq 2.5$ [23]. HOMA-B was calculated according to the formula: $(2.9 \times$ fasting insulin [pmol/1])/(FPG [mmol/1] - 3.5) [23]. Beta cell dysfunction was defined as HOMA-B within the lowest quartile among all samples measured.

Covariates Information on sociodemographic characteristics, medical history, diabetes family history and lifestyle factors (e.g. smoking status, alcohol consumption and physical activity) was obtained by using standard questionnaires at baseline and follow-up. Body weight, height, waist circumference and BP were measured by experienced nurses according to a standard protocol at two time points. Three seated BP measurements at the non-dominant arm were made consecutively with a $1 \mathrm{~min}$ interval after $5 \mathrm{~min}$ of rest, using an automated electronic device (Omron HEM-752 Fuzzy; Omron, Dalian, China). The three readings were averaged for analysis. BMI was calculated as body weight in kilograms divided by height in metres squared. Overweight or obese was defined as BMI $\geq 24 \mathrm{~kg} / \mathrm{m}^{2}$, as proposed by the Working Group on Obesity in China [24].

Statistical analysis Baseline sociodemographic and clinical characteristics of the participants were described according to urinary BPA concentrations. The data are presented as mean $\pm \mathrm{SD}$ or median (interquartile range [IQR]) values for continuous variables and frequencies for categorical variables. The measures of urinary BPA and blood metabolic markers at baseline and follow-up were compared using Wilcoxon signed-rank tests. In repeated-measures analyses, linear mixed 
models that included subject-specific random intercepts were used to evaluate the association between urinary BPA and markers of glucose homeostasis. To achieve normality assumption, both the values of BPA and metabolic markers were $\log _{10}$-transformed, and the regression coefficient estimates were subsequently converted to the per cent changes in these markers associated with a tenfold increase in urinary BPA concentrations. We also fitted generalised additive mixed models (GAMMs) using the $\mathrm{R} m g c v$ package (version 1.828, R Foundation for Statistical Computing, Vienna, Austria) to explore the departures from linearity in exposure-response relationships. For each model, repeated measures of metabolic markers were regressed on a penalised spline of urinary BPA concentrations. In consideration of the possible sex-specific effects of BPA on glucose homeostasis, analyses were conducted separately in men and women.

In addition to modelling glucose metabolic markers as continuous outcomes, logistic regression models with generalised estimating equations were used to estimate the ORs and 95\% CIs for fasting and postprandial hyperglycaemia, insulin resistance and beta cell dysfunction associated with urinary BPA concentrations. For repeated-measures analyses, the multivariable adjustment included age (years), urinary creatinine $(\mu \mathrm{mol} / \mathrm{l})$, education (primary school or less, middle school, or high school or higher), current cigarette smoking (yes/no), current alcohol consumption (yes/no), physical activity (none, light, or moderate to vigorous), menopausal status (yes/no, women only), systolic BP ( $\mathrm{mmHg})$, total cholesterol ( $\mathrm{mmol} / \mathrm{l})$ and family history of diabetes (yes/no). We additionally adjusted for BMI in the final model. Information on all covariates except for education and family history of diabetes was updated during follow-up and modelled as time-varying variables.

We evaluated potential effect modification by stratified analyses according to menopausal (premenopausal or postmenopausal) and BMI status (normal weight or overweight/obese). The significance of a multiplicative interaction term was determined using a likelihood ratio test. To address the potential bias from the loss to follow-up among several participants, we conducted a sensitivity analysis weighting the observations by the inverse probability for participation in the follow-up survey. To minimise the potential impact of extreme outliers of BPA measurements, we conducted an additional analysis by excluding participants who were in the top 5\% of urinary BPA concentrations. All analyses were performed using SAS (version 9.3; SAS Institute, Cary, NC, USA) and R software (version 3.5.3; R Foundation for Statistical Computing). A two-sided $p$ value $<0.05$ was considered statistically significant.

\section{Results}

The mean age of the study participants $(62.8 \%$ women) was 59.5 (SD 9.8) years. Generally, women were younger, had lower levels of education, were more likely to be neversmokers and drinkers, and to have a family history of diabetes. Sociodemographic and clinical characteristics of the participants by tertiles of urinary BPA concentrations are shown in Table 1. At baseline, participants with higher urinary BPA concentrations were younger, more educated and more likely to be current smokers. In women, those with higher concentrations of urinary BPA seemed to have lower HOMA-B, but the opposite tendency was observed in men.

The detection rate of BPA in all urine samples was $89.6 \%$, with a median concentration of 0.93 (IQR $0.53-1.61$ ) $\mu \mathrm{g} / \mathrm{l}$. Urinary concentrations of BPA were lower in women than in men. For glucose metabolic biomarkers, women had higher fasting insulin levels, HOMA-IR and HOMA-B compared with men, whereas no differences were found for FPG and OGTT 2 h PPG. Compared with the baseline measures, urinary BPA, FPG, OGTT $2 \mathrm{~h}$ PPG and HOMA-IR increased while fasting insulin levels and HOMA-B decreased in both sexes during a follow-up period of 4 years (all $p<0.05$ ) (ESM Table 1).

Relationships of urinary BPA concentrations with markers of glucose homeostasis are presented in Table 2 . In women, urinary BPA concentrations were positively associated with FPG and HOMA-IR and inversely associated with HOMA-B in the ageand urinary creatinine-adjusted model. Additional adjustment for potential confounders, including education, lifestyle, family history of diabetes and clinical variables, did not change the associations. When BMI was further included in the model, results were similar except for a remarkable attenuation of the association between BPA and HOMA-IR. Specifically, each tenfold increment in urinary BPA concentrations was associated with a $3.39 \%$ increase in FPG $(95 \%$ CI $2.24 \%, 4.55 \%)$ and an $11.6 \%$ decrease in HOMA-B $(95 \% \mathrm{CI}-15.8 \%,-7.18 \%)$ after full adjustment. However, urinary BPA concentrations were not associated with any markers of glucose homeostasis in men. Tests for interaction between BPA and sex were marginally significant for FPG $\left(p_{\text {interaction }}=0.06\right)$ and insulin levels $\left(p_{\text {interaction }}=0.05\right)$ and significant for HOMA-B $\left(p_{\text {interaction }}=0.005\right)$. Results from GAMM models confirmed the linearity of the observed associations with FPG and HOMA-B in women (Fig. 1a, b).

We then examined the associations of urinary BPA concentrations with metabolic markers categorised by clinically relevant thresholds (Table 3). There was a similar pattern of heterogeneity between sexes. BPA was significantly correlated with fasting hyperglycaemia and beta cell dysfunction in women but not in men, although the tests for interaction did not reach significance. After adjustment for covariates including BMI, the ORs associated with a tenfold increase in urinary BPA were 1.37 (95\% CI 1.10, 1.72) for fasting hyperglycaemia and 1.30 $(95 \%$ CI $1.02,1.65)$ for beta cell dysfunction.

The association between BPA and glucose homeostasis persisted irrespective of menopausal status in women (ESM Table 2). When stratified by BMI status, the estimates for 
Table 1 Baseline sociodemographic and clinical characteristics of the study population by tertiles of urinary BPA concentrations

\begin{tabular}{|c|c|c|c|c|c|c|}
\hline \multirow[t]{2}{*}{ Characteristic } & \multicolumn{3}{|l|}{ Women $(n=1467)$} & \multicolumn{3}{|l|}{ Men $(n=869)$} \\
\hline & Tertile 1 & Tertile 2 & Tertile 3 & Tertile 1 & Tertile 2 & Tertile 3 \\
\hline Participants, $n$ & 489 & 492 & 486 & 290 & 291 & 288 \\
\hline Age, years & $60.6 \pm 10.2$ & $58.9 \pm 9.5$ & $56.4 \pm 8.9$ & $63.4 \pm 9.9$ & $60.9 \pm 9.5$ & $58.8 \pm 9.0$ \\
\hline BMI, $\mathrm{kg} / \mathrm{m}^{2}$ & $24.7 \pm 3.4$ & $25.2 \pm 3.9$ & $25.1 \pm 3.6$ & $24.5 \pm 3.1$ & $24.7 \pm 3.2$ & $24.9 \pm 3.2$ \\
\hline $\begin{array}{l}\text { High school education or } \\
\text { higher, } n(\%)\end{array}$ & $119(25.1)$ & $134(27.7)$ & $137(28.8)$ & $91(31.6)$ & $99(35.1)$ & $97(34.9)$ \\
\hline $\begin{array}{l}\text { Moderate to vigorous } \\
\text { activity, } n(\%)\end{array}$ & $138(28.4)$ & $166(33.9)$ & $140(29.0)$ & $97(33.5)$ & $88(30.3)$ & $85(29.9)$ \\
\hline Current smoker, $n(\%)$ & $6(1.2)$ & $14(2.8)$ & $11(2.3)$ & $141(48.6)$ & $155(53.3)$ & $157(54.5)$ \\
\hline Current drinker, $n(\%)$ & $21(4.3)$ & $18(3.7)$ & $18(3.7)$ & $86(29.7)$ & $120(41.2)$ & $101(35.1)$ \\
\hline $\begin{array}{r}\text { Family history of } \\
\text { diabetes, } n(\%)\end{array}$ & $81(16.6)$ & $71(14.4)$ & $87(17.9)$ & $31(10.7)$ & $32(11.0)$ & $37(12.8)$ \\
\hline Menopause, $n(\%)$ & $392(82.4)$ & $403(83.3)$ & $355(74.3)$ & NA & NA & NA \\
\hline Waist circumference, $\mathrm{cm}$ & $83.9 \pm 9.5$ & $85.1 \pm 9.9$ & $84.7 \pm 10.1$ & $87.6 \pm 8.5$ & $87.7 \pm 8.7$ & $88.1 \pm 8.5$ \\
\hline Systolic BP, mmHg & $136.1 \pm 21.4$ & $134.5 \pm 21.9$ & $130.7 \pm 20.1$ & $140.3 \pm 22.3$ & $137.1 \pm 19.9$ & $133.8 \pm 19.3$ \\
\hline Diastolic BP, mmHg & $76.8 \pm 10.0$ & $76.1 \pm 9.5$ & $76.6 \pm 9.6$ & $80.2 \pm 11.4$ & $80.3 \pm 9.8$ & $79.6 \pm 9.7$ \\
\hline Total cholesterol, mmol/l & $5.3 \pm 1.0$ & $5.4 \pm 1.0$ & $5.2 \pm 0.9$ & $4.8 \pm 0.9$ & $4.8 \pm 0.9$ & $4.8 \pm 0.8$ \\
\hline LDL-cholesterol, mmol/1 & $2.4 \pm 0.7$ & $2.4 \pm 0.7$ & $2.4 \pm 0.7$ & $2.4 \pm 0.7$ & $2.3 \pm 0.7$ & $2.3 \pm 0.6$ \\
\hline HDL-cholesterol, $\mathrm{mmol} / \mathrm{l}$ & $1.5 \pm 0.3$ & $1.5 \pm 0.3$ & $1.4 \pm 0.3$ & $1.3 \pm 0.3$ & $1.2 \pm 0.2$ & $1.2 \pm 0.3$ \\
\hline Triacylglycerol, mmol/1 & $1.4(1.0-1.9)$ & $1.4(1.0-1.9)$ & $1.3(1.0-1.8)$ & $1.3(0.9-2.0)$ & $1.4(0.9-1.9)$ & $1.3(0.9-2.0)$ \\
\hline $\mathrm{FPG}, \mathrm{mmol} / \mathrm{l}$ & $4.9(4.6-5.4)$ & $5.0(4.7-5.3)$ & $5.0(4.7-5.3)$ & $5.0(4.6-5.4)$ & $5.0(4.6-5.3)$ & $4.9(4.6-5.3)$ \\
\hline OGTT 2 h PPG, mmol/1 & $6.8(5.6-8.1)$ & $6.7(5.7-8.0)$ & $6.8(5.8-7.9)$ & $7.0(5.7-8.4)$ & $6.9(5.6-8.0)$ & $7.0(5.7-8.3)$ \\
\hline Fasting insulin, pmol/1 & $48.3(32.3-69.5)$ & $49.0(33.5-70.4)$ & $47.2(32.9-69.8)$ & $40.2(27.1-57.9)$ & $41.9(27.0-62.0)$ & $43.7(25.3-60.6)$ \\
\hline HOMA-IR & $1.5(1.0-2.4)$ & $1.5(1.0-2.3)$ & $1.6(1.0-2.3)$ & $1.3(0.8-1.9)$ & $1.4(0.8-1.9)$ & $1.4(0.8-2.0)$ \\
\hline HOMA-B & $95.8(63.2-143.5)$ & $94.9(63.4-142.8)$ & $91.5(65.9-137.3)$ & $77.8(52.0-120.0)$ & $82.6(52.1-128.4)$ & $82.8(52.5-129.7)$ \\
\hline
\end{tabular}

Values are presented as mean $\pm \mathrm{SD}$ or median (IQR) unless otherwise indicated

Urinary BPA tertiles in women: T1 $(<0.53 \mu \mathrm{g} / \mathrm{l}), \mathrm{T} 2(0.53-1.08 \mu \mathrm{g} / \mathrm{l}), \mathrm{T} 3(>1.08 \mu \mathrm{g} / \mathrm{l})$; urinary BPA tertiles in men: T1 $(<0.67 \mu \mathrm{g} / \mathrm{l}), \mathrm{T} 2(0.67-1.29 \mu \mathrm{g} / \mathrm{l})$, T3 $(>1.29 \mu \mathrm{g} / \mathrm{l})$

There were 54 missing values for education (33 women, 21 men), 13 for physical activity ( 8 women, 5 men) and 29 for menopause status

NA, not applicable

HOMA-B were stronger in overweight/obese women (change $-13.7 \%$; $95 \% \mathrm{CI}-19.3 \%,-7.61 \%$ ) vs those with normal weight (change $-6.74 \% ; 95 \% \mathrm{CI}-13.2 \%, 0.20 \%)\left(p_{\text {interaction }}=0.07\right)$ (Fig. 2a). Conversely, BPA was predominantly associated with HOMA-IR in normal-weight women (Fig. 2a). Additionally, there seemed to be a divergent association with fasting insulin between the two subgroups $\left(p_{\text {interaction }}=0.04\right.$ ) (Fig. $\left.2 \mathrm{a}\right)$. We did not observe any significant differences in markers of glucose homeostasis with BPA across BMI categories in men (Fig. 2b).

The observed associations were robust in multiple sensitivity analyses. First, the analyses that weighted the observations by the inverse probability for successful follow-up yielded similar results (ESM Table 3). Second, the associations between BPA and glucose homeostasis remained and became more prominent when we excluded the participants in the top $5 \%$ of urinary BPA concentrations (ESM Table 4).

\section{Discussion}

In this repeated-measures-based, longitudinal study among middle-aged and elderly adults without baseline diabetes, we found a sex-specific association between urinary BPA and glucose homeostasis. In women, BPA was positively associated with FPG and inversely associated with HOMA-B in a dose-response manner. The estimates were stronger among overweight or obese women than among those who were of normal weight. Moreover, elevated BPA was associated in women with an increased risk of having fasting hyperglycaemia and beta cell dysfunction. We found no evidence of associations between BPA and glucose metabolic outcomes in men. These results demonstrate that female exposure to BPA may be a risk factor for disturbance of glucose homeostasis even before the development of diabetes.

Despite the controversial results, the past several years have seen growing of evidence linking BPA to diabetes risk 
Table 2 Adjusted per cent changes $(95 \% \mathrm{CI})$ in markers of glucose homeostasis associated with a tenfold increase in urinary BPA concentrations

\begin{tabular}{|c|c|c|c|c|c|}
\hline \multirow[t]{2}{*}{ Variable } & \multicolumn{2}{|l|}{ Women } & \multicolumn{2}{|l|}{ Men } & \multirow[t]{2}{*}{$p_{\text {interactiol }}$} \\
\hline & $\begin{array}{l}\text { Per cent change } \\
(95 \% \mathrm{CI})\end{array}$ & $p$ value & $\begin{array}{l}\text { Per cent change } \\
(95 \% \mathrm{CI})\end{array}$ & $p$ value & \\
\hline \multicolumn{6}{|l|}{$\mathrm{FPG}, \mathrm{mmol} / \mathrm{l}$} \\
\hline Age- and creatinine-adjusted & $4.21(3.01,5.42)$ & $<0.001$ & $-0.25(-1.92,1.46)$ & 0.77 & 0.009 \\
\hline Multivariable-adjusted $^{\mathrm{a}}$ & $3.60(2.45,4.77)$ & $<0.001$ & $0.02(-1.64,1.71)$ & 0.98 & 0.054 \\
\hline Multivariable- and BMI-adjusted & $3.39(2.24,4.55)$ & $<0.001$ & $-0.11(-1.77,1.57)$ & 0.89 & 0.06 \\
\hline \multicolumn{6}{|l|}{ OGTT 2 h PPG, mmol/1 } \\
\hline Age- and creatinine-adjusted & $2.18(-0.002,4.42)$ & 0.05 & $-0.59(-4.28,3.25)$ & 0.76 & 0.22 \\
\hline Multivariable-adjusted & $2.08(-0.10,4.30)$ & 0.06 & $0.03(-3.68,3.87)$ & 0.99 & 0.39 \\
\hline Multivariable- and BMI-adjusted & $1.41(-0.73,3.59)$ & 0.20 & $-0.38(-4.04,3.42)$ & 0.84 & 0.41 \\
\hline \multicolumn{6}{|l|}{ Fasting insulin, pmol/1 } \\
\hline Age- and creatinine-adjusted & $1.35(-2.66,5.52)$ & 0.51 & $3.38(-3.09,10.3)$ & 0.31 & 0.36 \\
\hline Multivariable-adjusted & $1.26(-2.80,5.49)$ & 0.55 & $5.36(-1.22,12.4)$ & 0.11 & 0.15 \\
\hline Multivariable- and BMI-adjusted & $-1.99(-5.69,1.86)$ & 0.31 & $3.92(-2.14,10.3)$ & 0.21 & 0.05 \\
\hline \multicolumn{6}{|l|}{ HOMA-IR } \\
\hline Age- and creatinine-adjusted & $6.21(1.63,11.0)$ & 0.008 & $4.02(-2.89,11.4)$ & 0.26 & 0.79 \\
\hline Multivariable-adjusted & $5.17(0.61,9.94)$ & 0.03 & $6.29(-0.68,13.8)$ & 0.08 & 0.31 \\
\hline Multivariable- and BMI-adjusted & $1.52(-2.62,5.83)$ & 0.48 & $4.21(-2.13,11.0)$ & 0.20 & 0.16 \\
\hline \multicolumn{6}{|l|}{ HOMA-B } \\
\hline Age- and creatinine-adjusted & $-11.2(-15.6,-6.63)$ & $<0.001$ & $5.01(-3.49,14.2)$ & 0.26 & 0.009 \\
\hline Multivariable-adjusted & $-9.24(-13.7,-4.56)$ & $<0.001$ & $6.04(-2.59,15.4)$ & 0.18 & 0.02 \\
\hline Multivariable- and BMI-adjusted & $-11.6(-15.8,-7.18)$ & $<0.001$ & $4.82(-3.44,13.8)$ & 0.26 & 0.005 \\
\hline
\end{tabular}

${ }^{\mathrm{a}}$ Linear mixed models were adjusted for age, urinary creatinine, education, smoking status, drinking status, physical activity, menopausal status (women only), systolic BP, total cholesterol and family history of diabetes. All covariates with the exception of education and family history of diabetes were modelled as time-varying variables

in humans [13-16, 25]. However, data on the effect of BPA exposure on glucose metabolic traits have been limited. Several previous studies using a nationally representative sample have reported cross-sectionally positive associations between urinary BPA and indices of glucose metabolism, including blood glucose and insulin, HOMA-IR and HOMA-B $[17,18]$. Our previous study in the same cohort also indicated that urinary BPA was associated with longitudinal change in FPG among adults with a high genetic predisposition to diabetes [20]. The present study is unique in that we systematically assessed BPA exposure in relation to markers of glucose metabolism based on a repeated-measures analysis over an extended period. We found consistent associations of urinary BPA with increased FPG and reduced HOMA-B, and the effects were dependent on sex. Given the high within-person variations of BPA concentrations [19, 26], exposure misclassification may exist if quantification of exposure level is based on a single measurement from one spot urine sample, which has usually been recognised as a major limitation in previous epidemiological studies on BPA-related health outcomes. In this study, the longitudinal design with combined repeated measures of BPA and glucose metabolic traits enabled us to obtain a more robust assessment of the association, even though urinary BPA concentrations in our population were generally lower than those reported in other populations [17, 18].

Another important aspect that merits notice is that previous studies exploring the association between BPA and glucose homeostasis included diabetic individuals $[17,18]$. The reported associations may be partly biased due to the influence of diet and/or lifestyle changes on BPA exposure after diagnosis of diabetes. Meanwhile, the link between BPA and glucose homeostasis among non-diabetic individuals remains unclear. In agreement with our findings, a prior study among US adults free of diabetes found that urinary BPA was associated with prediabetes in women [27]. The current study adds to the existing evidence by demonstrating for the first time that female exposure to BPA was associated with impaired glucose homeostasis prior to the onset of diabetes, which may have important clinical and public health implications for developing intervention strategies, especially for a high-risk population, to help curb the epidemic of diabetes.

The sex-specific association between BPA and glucose homeostasis in this study is presumably related to the differential modulation of glucose metabolism by endogenous sex hormones in men and women [28, 29]. In view of the oestrogenic property of BPA, it seems reasonable to assume an interaction 


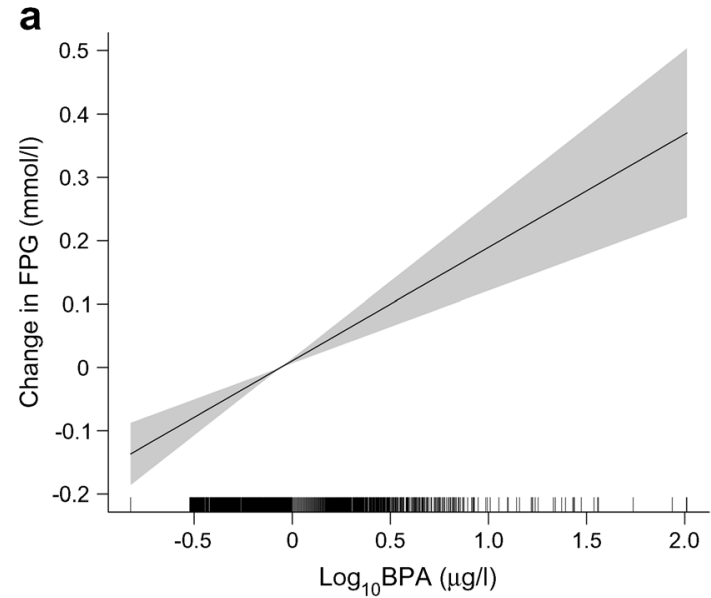

Fig. 1 Dose-response curves for the associations of urinary BPA concentrations with FPG (a) and HOMA-B (b) in women. GAMMs with penalised regression splines were adjusted for age, urinary creatinine, education, smoking status, drinking status, physical activity, menopausal status, systolic BP, total cholesterol, BMI and family history of diabetes.

between systemic concentrations of endogenous sex hormones and BPA for metabolic disorders. There is also a possibility that the divergent associations may reflect sex differences in behaviours and lifestyle factors that were not captured b

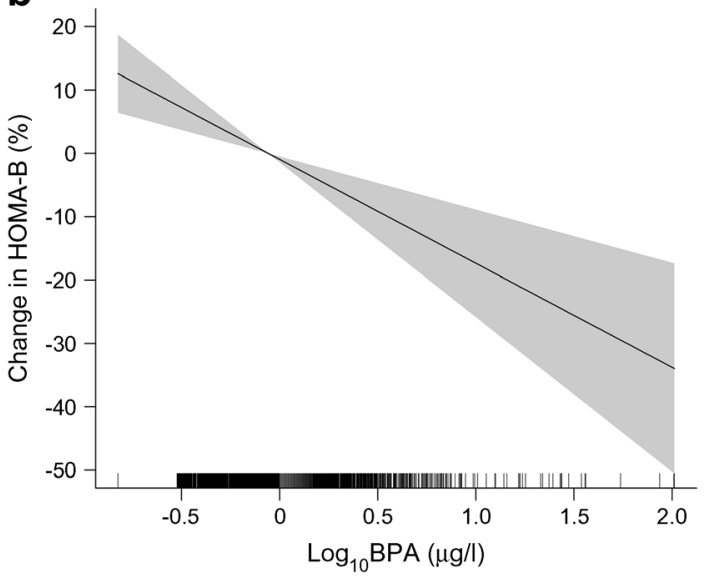

All covariates, with the exception of education and family history of diabetes, were modelled as time-varying variables. Short vertical lines located on the $x$-axes of each plot indicate the values at which observations were made. Shaded areas indicate 95\% CIs. Linearity of the observed associations was confirmed

Table 3 Adjusted ORs (95\% CI) of fasting and postprandial hyperglycaemia, insulin resistance and beta cell dysfunction associated with a tenfold increase in urinary BPA concentrations

\begin{tabular}{|c|c|c|c|c|c|}
\hline \multirow[t]{2}{*}{ Variable } & \multicolumn{2}{|l|}{ Women } & \multicolumn{2}{|l|}{ Men } & \multirow[t]{2}{*}{$p_{\text {interaction }}$} \\
\hline & OR $(95 \% \mathrm{CI})$ & $p$ value & OR $(95 \% \mathrm{CI})$ & $p$ value & \\
\hline \multicolumn{6}{|l|}{ Fasting hyperglycaemia } \\
\hline Age- and creatinine-adjusted & $1.46(1.18,1.80)$ & $<0.001$ & $0.97(0.71,1.33)$ & 0.84 & 0.15 \\
\hline Multivariable-adjusted $^{\mathrm{a}}$ & $1.42(1.14,1.77)$ & 0.002 & $0.96(0.70,1.33)$ & 0.83 & 0.24 \\
\hline Multivariable- and BMI-adjusted & $1.37(1.10,1.72)$ & 0.006 & $0.95(0.68,1.31)$ & 0.74 & 0.26 \\
\hline \multicolumn{6}{|l|}{ Postprandial hyperglycaemia } \\
\hline Age- and creatinine-adjusted & $0.99(0.82,1.21)$ & 0.95 & $1.03(0.77,1.36)$ & 0.86 & 0.90 \\
\hline Multivariable-adjusted & $0.96(0.78,1.17)$ & 0.66 & $1.07(0.79,1.44)$ & 0.67 & 0.58 \\
\hline Multivariable- and BMI-adjusted & $0.92(0.75,1.13)$ & 0.41 & $1.05(0.78,1.42)$ & 0.75 & 0.55 \\
\hline \multicolumn{6}{|l|}{ Insulin resistance } \\
\hline Age- and creatinine-adjusted & $1.03(0.84,1.26)$ & 0.80 & $1.07(0.73,1.57)$ & 0.73 & 0.96 \\
\hline Multivariable-adjusted & $1.01(0.82,1.25)$ & 0.90 & $1.21(0.80,1.83)$ & 0.37 & 0.58 \\
\hline Multivariable- and BMI-adjusted & $0.89(0.71,1.13)$ & 0.34 & $1.20(0.77,1.86)$ & 0.42 & 0.29 \\
\hline \multicolumn{6}{|l|}{ Beta cell dysfunction } \\
\hline Age- and creatinine-adjusted & $1.21(0.96,1.51)$ & 0.10 & $0.85(0.64,1.12)$ & 0.26 & 0.17 \\
\hline Multivariable-adjusted & $1.15(0.91,1.44)$ & 0.25 & $0.81(0.61,1.08)$ & 0.15 & 0.14 \\
\hline Multivariable- and BMI-adjusted & $1.30(1.02,1.65)$ & 0.03 & $0.85(0.63,1.16)$ & 0.30 & 0.09 \\
\hline
\end{tabular}

Fasting hyperglycaemia: $\geq 5.6 \mathrm{mmol} / \mathrm{l}$; postprandial hyperglycaemia: $\geq 7.8 \mathrm{mmol} / \mathrm{l}$; insulin resistance: HOMA-IR $\geq 2.5$; beta cell dysfunction: HOMA-B within the lowest quartile among all samples measured

${ }^{a}$ Logistic regression models with generalised estimating equations were adjusted for age, urinary creatinine, education, smoking status, drinking status, physical activity, menopausal status (women only), systolic BP, total cholesterol and family history of diabetes. All covariates with the exception of education and family history of diabetes were modelled as time-varying variables 

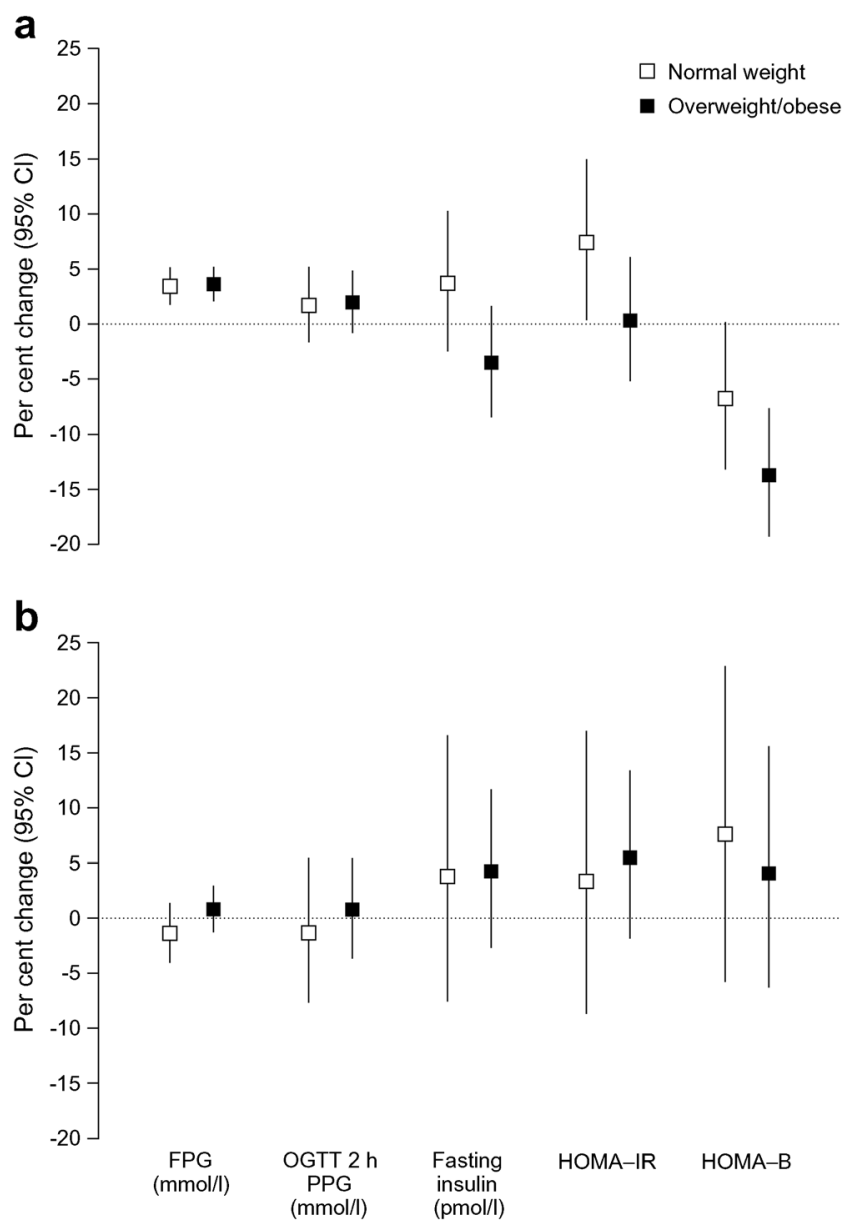

Fig. 2 Adjusted per cent changes $(95 \% \mathrm{CI})$ in markers of glucose homeostasis associated with a tenfold increase in urinary BPA concentration stratified by baseline BMI status (normal weight [BMI $<24 \mathrm{~kg} / \mathrm{m}^{2}$ ] or overweight/obese $\left[\right.$ BMI $\left.\geq 24 \mathrm{~kg} / \mathrm{m}^{2}\right]$ ) in women (a) and men (b). The linear mixed models were adjusted for age, urinary creatinine, education, smoking status, drinking status, physical activity, menopausal status (women only), systolic BP, total cholesterol and family history of diabetes. All covariates, with the exception of education and family history of diabetes, were modelled as time-varying variables. Tests for interaction between BPA and BMI status were significant for fasting insulin $\left(p_{\text {interaction }}=0.04\right)$ and close to significance for HOMA-IR and HOMA-B (both $p_{\text {interaction }}=$ 0.07 ) in women

cell function has been documented in animal experiments $[10,30,31]$. In our analysis, we detected a significant increase in FPG and decline in beta cell function among women, especially among those who were overweight or obese. Likewise, BPA was reported to suppress the viability of rat insulinoma (INS-1) cells and disturb glucosestimulated insulin secretion by triggering beta cell apoptosis through damage of mitochondria [32]. Studies using pancreatic islet models also indicated the role of BPA in the process of impaired insulin secretion and beta cell failure [33, 34]. In overweight or obese individuals, the body's demand for insulin markedly increases, and beta cell failure is more likely to occur owing to inadequate expansion of the beta cell mass and/or failure of the beta cells in response to glucose [35]. Taken together, our findings suggest that BPA has the potential to disturb glucose homeostasis by inducing beta cell dysfunction, which may be aggravated by overweight or obesity.

A major strength of this study is the longitudinal design with repeated measurements of both exposure and outcome biomarkers as well as covariates, which provided the unique statistical modelling to more precisely detect the association of glucose homeostasis with subtle BPA exposure. In addition, our study focused on non-diabetic individuals, which not only minimised the potential biases from disease treatments or diet and lifestyle changes after diabetes diagnosis, but also offered novel findings to reveal the influence of BPA on glucose homeostasis when blood glucose levels were still within the relatively normal range.

Several limitations should be acknowledged. First, although we controlled for an array of important covariates, the possibility of unmeasured or residual confounding by other sources associated with BPA exposure and glucose homeostasis cannot be excluded. Second, missing follow-up data of several participants might have introduced bias into the final estimates. However, results from the sensitivity analysis weighting the observations by the inverse probability for participation in the follow-up survey suggested that bias was unlikely. Third, the current study was conducted among a middle-aged and elderly Chinese population with an average age of $\sim 60$ years; hence, the results may not be directly generalisable to younger and other ethnic populations. Fourth, since we collected blood samples just at $0 \mathrm{~h}$ and $2 \mathrm{~h}$ during the OGTT, a dynamic assessment of beta cell function such as the insulinogenic index was unavailable. Finally, the role of other environmental chemicals that have similar effects cannot be determined. For example, exposure to phthalates has been reported to associate with disturbance of glucose metabolism and diabetes [36, 37]. Similar to BPA, phthalates are a large group of industrial high-volume chemicals that are used as plasticisers in various consumer products. Widespread exposure to phthalates leads to consistent detection of their metabolites in human biological samples [38]. Therefore, the impact of phthalate and other correlated chemicals from similar sources remains to be clarified.

In conclusion, we found that elevated concentrations of urinary BPA were associated with increased FPG and reduced HOMA-B, as well as a higher risk of having fasting hyperglycaemia and beta cell dysfunction, among nondiabetic women. Our findings suggest that BPA, a commonly used chemical, might impair glucose homeostasis before diabetes onset at the exposure level in the general middle-aged and elderly population, which highlights the importance of intervention strategies against BPA exposure to help prevent and alleviate the burdens of diabetes. 
Acknowledgements The investigators are grateful to all participants for their cooperation in the study.

Data availability Data are available from the authors on request.

Funding This work was funded by the Chinese Ministry of Finance, the 973 Foundation (grant 2015CB553601), National Key R\&D Program of China (grants 2016YFC1305600, 2017YFC1310700, 2016YFC0901200 and 2016YFC1304904), National Natural Science Foundation of China (grants 81622011 and 81621061), Shanghai Pujiang Program (18PJ1409600) and Shanghai Municipal Education CommissionGaofeng Clinical Medicine and Doctoral Innovation Grant Support from Shanghai Jiao Tong University School of Medicine (grants 20171901, 20161301, 20152508, 20161307 and BXJ201908).

Duality of interest The authors declare that there is no duality of interest associated with this manuscript.

Contribution statement BW, ML, TW, YB and GN contributed to the study design and concept. BW and ML analysed the data and drafted the manuscript. ZZ, JL, YC, YX, MX, WW and GN contributed to data interpretation and the editing of the manuscript. TW and YB critically revised the manuscript for important intellectual content. All authors have approved the final version to be published. TW, YB and GN guarantee this work and have full access to all of the data and take responsibility for the integrity of the data.

\section{References}

1. GBD 2015 Disease and Injury Incidence and Prevalence Collaborators (2016) Global, regional, and national incidence, prevalence, and years lived with disability for 310 diseases and injuries, 1990-2015: a systematic analysis for the Global Burden of Disease Study 2015. Lancet 388(10053):1545-1602. https://doi. org/10.1016/S0140-6736(16)31678-6

2. Qi L, Hu FB, Hu G (2008) Genes, environment, and interactions in prevention of type 2 diabetes: a focus on physical activity and lifestyle changes. Curr Mol Med 8(6):519-532. https://doi.org/10. 2174/156652408785747915

3. Casals-Casas C, Desvergne B (2011) Endocrine disruptors: from endocrine to metabolic disruption. Annu Rev Physiol 73(1):135162. https://doi.org/10.1146/annurev-physiol-012110-142200

4. Ruiz D, Becerra M, Jagai JS, Ard K, Sargis RM (2018) Disparities in environmental exposures to endocrine-disrupting chemicals and diabetes risk in vulnerable populations. Diabetes Care 41(1):193205. https://doi.org/10.2337/dc16-2765

5. Hao M, Ding L, Xuan L et al (2018) Urinary bisphenol A concentration and the risk of central obesity in Chinese adults: a prospective study. J Diabetes 10(6):442-448. https://doi.org/10.1111/17530407.12531

6. Ehrlich S, Calafat AM, Humblet O, Smith T, Hauser R (2014) Handling of thermal receipts as a source of exposure to bisphenol A. JAMA 311(8):859-860. https://doi.org/10.1001/jama.2013. 283735

7. Lakind JS, Naiman DQ (2008) Daily intake of bisphenol A and potential sources of exposure: 2005-2006 National Health and Nutrition Examination Survey. J Expo Sci Environ Epidemiol 21(3):272-279. https://doi.org/10.1038/jes.2010.9

8. Dekant W, Volkel W (2008) Human exposure to bisphenol A by biomonitoring: methods, results and assessment of environmental exposures. Toxicol Appl Pharmacol 228(1):114-134. https://doi. org/10.1016/j.taap.2007.12.008
9. Vandenberg LN, Chahoud I, Heindel JJ, Padmanabhan V, Paumgartten FJ, Schoenfelder G (2010) Urinary, circulating, and tissue biomonitoring studies indicate widespread exposure to bisphenol A. Environ Health Perspect 118(8):1055-1070. https:// doi.org/10.1289/ehp.0901716

10. Alonso-Magdalena P, Morimoto S, Ripoll C, Fuentes E, Nadal A (2006) The estrogenic effect of bisphenol A disrupts pancreatic $\beta$ cell function in vivo and induces insulin resistance. Environ Health Perspect 114(1):106-112. https://doi.org/10.1289/ehp.8451

11. Ben-Jonathan N, Hugo ER, Brandebourg TD (2009) Effects of bisphenol A on adipokine release from human adipose tissue: implications for the metabolic syndrome. Mol Cell Endocrinol 304(12):49-54. https://doi.org/10.1016/j.mce.2009.02.022

12. Bindhumol V, Chitra KC, Mathur PP (2003) Bisphenol A induces reactive oxygen species generation in the liver of male rats. Toxicology 188(2-3):117-124. https://doi.org/10.1016/S0300483X(03)00056-8

13. Lang IA, Galloway TS, Scarlett A et al (2008) Association of urinary bisphenol A concentration with medical disorders and laboratory abnormalities in adults. JAMA 300(11):1303-1310. https:// doi.org/10.1001/jama.300.11.1303

14. LaKind JS, Goodman M, Naiman DQ (2012) Use of NHANES data to link chemical exposures to chronic diseases: a cautionary tale. PLoS One 7(12):e51086. https://doi.org/10.1371/journal.pone. 0051086

15. Shankar A, Teppala S (2011) Relationship between urinary bisphenol A levels and diabetes mellitus. J Clin Endocrinol Metab 96(12):3822-3826. https://doi.org/10.1210/jc.2011-1682

16. Ning G, Bi Y, Wang T et al (2011) Relationship of urinary bisphenol A concentration to risk for prevalent type 2 diabetes in Chinese adults: a cross-sectional analysis. Ann Intern Med 155(6):368 374. https://doi.org/10.7326/0003-4819-155-6-201109200-00005

17. Tai X, Chen Y (2016) Urinary bisphenol A concentrations positively associated with glycated hemoglobin and other indicators of diabetes in Canadian men. Environ Res 147:172-178. https://doi. org/10.1016/j.envres.2016.02.006

18. Beydoun HA, Khanal S, Zonderman A, Beydoun MA (2014) Sex differences in the association of urinary bisphenol-A concentration with selected indices of glucose homeostasis among U.S. adults. Ann Epidemiol 24(2):90-97. https://doi.org/10.1016/j.annepidem. 2013.07.014

19. Ye X, Wong LY, Bishop AM, Calafat AM (2011) Variability of urinary concentrations of bisphenol A in spot samples, first morning voids, and 24-hour collections. Environ Health Perspect 119(7): 983-988. https://doi.org/10.1289/ehp.1002701

20. Bi Y, Wang W, Xu M et al (2016) Diabetes genetic risk score modifies effect of bisphenol A exposure on deterioration in glucose metabolism. J Clin Endocrinol Metab 101(1):143-150. https://doi. org/10.1210/jc.2015-3039

21. He Y, Miao M, Herrinton LJ et al (2009) Bisphenol A levels in blood and urine in a Chinese population and the personal factors affecting the levels. Environ Res 109(5):629-633. https://doi.org/ 10.1016/j.envres.2009.04.003

22. American Diabetes Association (2009) Diagnosis and classification of diabetes mellitus. Diabetes Care 32(Suppl 1):S62-S67. https:// doi.org/10.2337/dc09-S062

23. Matthews DR, Hosker JP, Rudenski AS, Naylor BA, Treacher DF, Turner RC (1985) Homeostasis model assessment: insulin resistance and beta-cell function from fasting plasma glucose and insulin concentrations in man. Diabetologia 28(7):412-419. https://doi.org/ 10.1007/BF00280883

24. Zhou BF (2002) Predictive values of body mass index and waist circumference for risk factors of certain related diseases in Chinese adults - study on optimal cut-off points of body mass index and waist circumference in Chinese adults. Biomed Environ Sci 15(1): 83-96 
25. Sun Q, Cornelis MC, Townsend MK et al (2014) Association of urinary concentrations of bisphenol $\mathrm{A}$ and phthalate metabolites with risk of type 2 diabetes: a prospective investigation in the Nurses' Health Study (NHS) and NHSII cohorts. Environ Health Perspect 122(6):616-623. https://doi.org/10.1289/ehp.1307201

26. Mahalingaiah S, Meeker JD, Pearson KR et al (2008) Temporal variability and predictors of urinary bisphenol A concentrations in men and women. Environ Health Perspect 116(2):173-178. https:// doi.org/10.1289/ehp. 10605

27. Sabanayagam C, Teppala S, Shankar A (2013) Relationship between urinary bisphenol A levels and prediabetes among subjects free of diabetes. Acta Diabetol 50(4):625-631. https://doi.org/10. 1007/s00592-013-0472-z

28. Ding EL, Song Y, Malik VS, Liu S (2006) Sex differences of endogenous sex hormones and risk of type 2 diabetes: a systematic review and meta-analysis. JAMA 295(11):1288-1299. https://doi. org/10.1001/jama.295.11.1288

29. Liu S, Sun Q (2018) Sex differences, endogenous sex-hormone hormones, sex-hormone binding globulin, and exogenous disruptors in diabetes and related metabolic outcomes. J Diabetes 10(6):428-441. https://doi.org/10.1111/1753-0407.12517

30. Alonso-Magdalena P, Ropero AB, Carrera MP et al (2008) Pancreatic insulin content regulation by the estrogen receptor ER $\alpha$. PLoS One 3(4):e2069. https://doi.org/10.1371/journal.pone.0002069

31. Ropero AB, Alonso-Magdalena P, García-García E, Ripoll C, Fuentes E, Nadal A (2008) Bisphenol-A disruption of the endocrine pancreas and blood glucose homeostasis. Int J Androl 31(2):194200. https://doi.org/10.1111/j.1365-2605.2007.00832.x

32. Lin Y, Sun X, Qiu L et al (2013) Exposure to bisphenol A induces dysfunction of insulin secretion and apoptosis through the damage of mitochondria in rat insulinoma (INS-1) cells. Cell Death Dis 4(1):e460. https://doi.org/10.1038/cddis.2012.206

33. Song L, Xia W, Zhou Z et al (2012) Low-level phenolic estrogen pollutants impair islets morphology and $\beta$-cells function in isolated rat islets. J Endocrinol 215(2):303-311. https://doi.org/10.1530/ JOE-12-0219

34. Bansal A, Rashid C, Xin F et al (2017) Sex- and dose-specific effects of maternal bisphenol A exposure on pancreatic islets of first- and second-generation adult mice offspring. Environ Health Perspect 125(9):097022. https://doi.org/10.1289/EHP1674

35. Rhodes CJ (2005) Type 2 diabetes - a matter of beta-cell life and death? Science 307(5708):380-384. https://doi.org/10.1126/ science. 1104345

36. Dales RE, Kauri LM, Cakmak S (2018) The associations between phthalate exposure and insulin resistance, $\beta$-cell function and blood glucose control in a population-based sample. Sci Total Environ 612:1287-1292. https://doi.org/10.1016/j.scitotenv.2017.09.009

37. Lind PM, Zethelius B, Lind L (2012) Circulating levels of phthalate metabolites are associated with prevalent diabetes in the elderly. Diabetes Care 35(7):1519-1524. https://doi.org/10.2337/dc112396

38. Zota AR, Calafat AM, Woodruff TJ (2014) Temporal trends in phthalate exposures: findings from the National Health and Nutrition Examination Survey, 2001-2010. Environ Health Perspect 122(3):235-241. https://doi.org/10.1289/ehp.1306681

Publisher's note Springer Nature remains neutral with regard to jurisdictional claims in published maps and institutional affiliations. 